\title{
Surface imaging with UHV SLEEM and SEM LEEM
}

\author{
Šárka Mikmeková1 ${ }^{*}$, Pavel Jánský ${ }^{2}$, Vladimír Kolařík ${ }^{2}$ and Ilona Müllerová1 \\ 1. ISI Brno, Czech Academy of Sciences, Brno, Czech Republic \\ 2. Delong Instruments a.s., Brno, Czech Republic \\ * Corresponding author: sarka@isibrno.cz
}

From fundamental studies in the materials science up to diagnostics of industrial materials a reliable method enabling characterization of surface properties with high sensitivity and high spatial resolution is urgently needed. Low energy electron microscopy method has proven itself eminently sensitive not only to the topography of the sample surface but also to its chemical composition, crystalline structure and electronic configuration [1, 2].

The specimen under investigation was graphene layers on polycrystalline $\mathrm{Cu}$ substrate. The specimen was characterized by means of two unique instrument, namely a UHV SLEEM developed at ISI Brno [3] and an SEM LEEM developed by Delong Instruments a.s. [4] (Fig. 1). Both instruments enable to observe a specimen surface at arbitrary low landing energies. In the case of the UHV SLEEM, the primary electrons are retarded to their final landing energy by the electrostatic field created between the negatively biased specimen and the earthed detector and the emitted electrons are collimated to the optical axis and accelerated towards the detector situated below the pole piece. The SEM LEEM has rotationally asymmetric imaging and detection system that provides separate beam lines of primary and signal beams. This arrangement enables to reach zero landing energy without loss of signal on the optical axis. As in the UHV SLEEM, the landing energy is controlled by the sample bias. The great advantage is that detected electrons can be separated which enables to distinguish between elastically scattered and secondary electrons.

Fig. 2 shows a series of the micrographs obtained by the UHV SLEEM (left) and the SEM LEEM (right). It is evident that the contrast between the graphene and the Cu substrate in the UHV SLEEM and the SEM LEEM micrographs obtained at the same landing electron energy is not similar. The energy dependence of the graphene/ $\mathrm{Cu}$ contrast is shown in Fig. 3. The contrast was calculated as $\mathrm{C}(\mathrm{A})=$ $\left(\mathrm{S}_{\mathrm{G}(\mathrm{A})}-\mathrm{S}_{\mathrm{A}}\right) /\left(\mathrm{S}_{\mathrm{G}(\mathrm{A})}+\mathrm{S}_{\mathrm{A}}\right) * 100$ [\%], where $\mathrm{S}_{\mathrm{G}(\mathrm{A})}$ is image signal averaged over the graphene situated on grain $A$ and $S_{A}$ is image signal averaged over the grain A (likewise: $C(B)=\left(S_{G(B)}-S_{B}\right) /\left(S_{G(B)}+S_{B}\right) *$ 100 [\%]). At energies above $30 \mathrm{eV}$, the UHV SLEEM and the SEM LEEM images have inverse contrast, which is probably caused by significant contribution of the in-elastic electrons to the signal collected by the UHV SLEEM. In the landing energy range of units of $\mathrm{eV}$, peaks are presented on the UHV SLEEM and the SEM LEEM contrast curves. The peak positions are specific for the instruments. This phenomenon will be discussed in more detail.

\section{References:}

[1] E. Bauer “Low Energy Electron Microscopy” (Springer Verlag, New York), 2014.

[2] I Mullerova et. al. Adv. Imag. Electron Phys. 128 (2003), p. 309-443.

[3] www.isibrno.cz

[4] www.delong.cz

[5] The authors acknowledge funding from the Technology Agency of the Czech Republic (Competence center: Electron microscopy, no: TE01020118). 

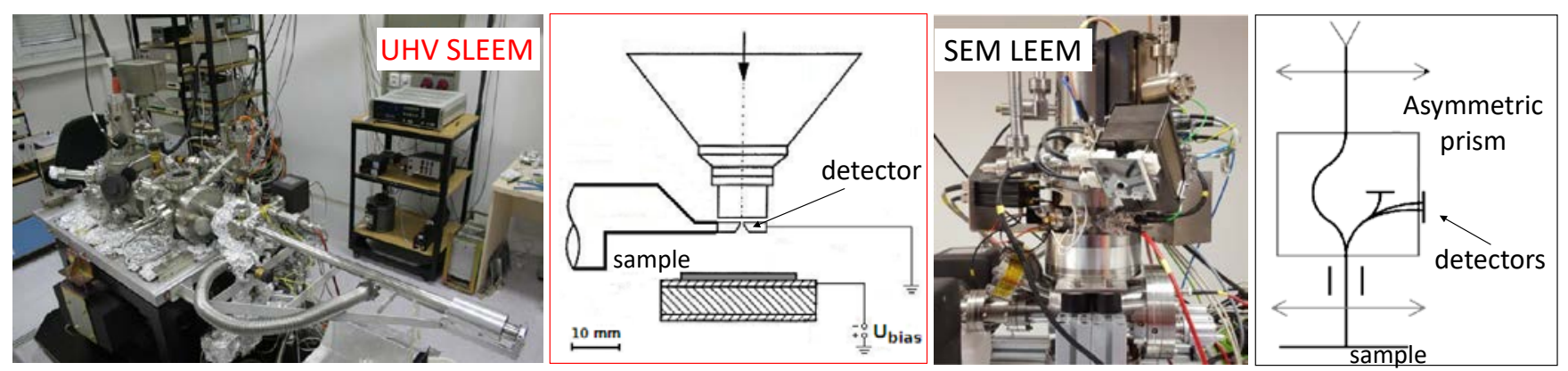

Figure 1. UHV SLEEM instrument and a schematic sketch of the cathode lens system (red border); SEM LEEM instrument together with its simplified schematic description (black border).
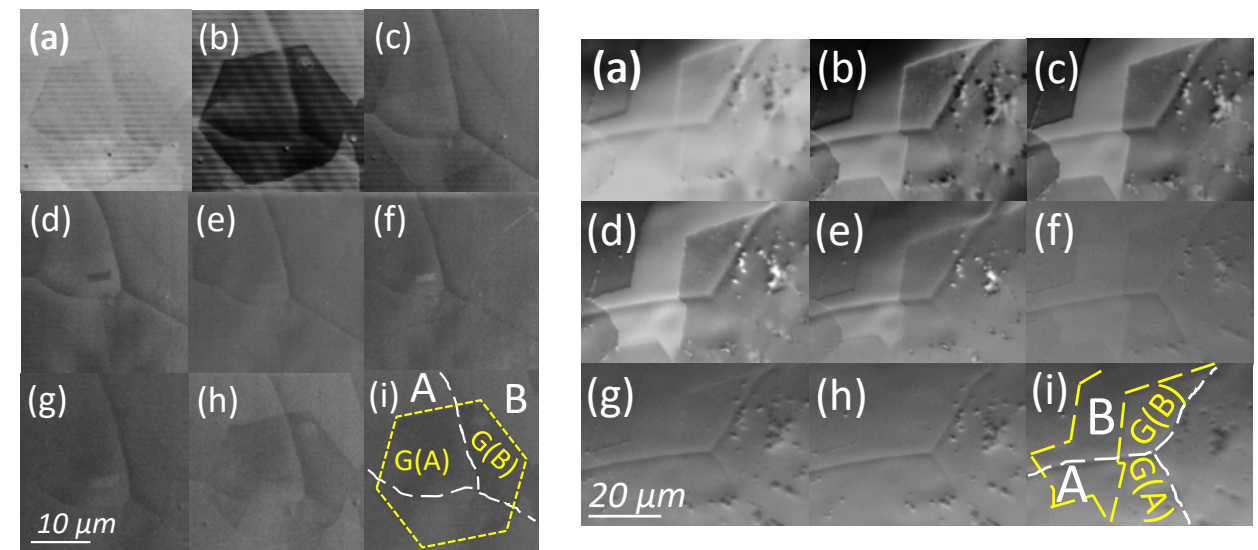

Figure 2. Micrographs of the graphene/Cu specimen obtained at energies: $1 \mathrm{eV}$ (a), $2 \mathrm{eV}$ (b), $3 \mathrm{eV}$ (c), $4 \mathrm{eV}$ (d), $5 \mathrm{eV}$ (e), $10 \mathrm{eV}$ (f), $15 \mathrm{eV}$ (g), $25 \mathrm{eV}$ (h) and $50 \mathrm{eV}$ (i). Left side: UHV SLEEM instrument micrographs, right side: SEM LEEM micrographs.

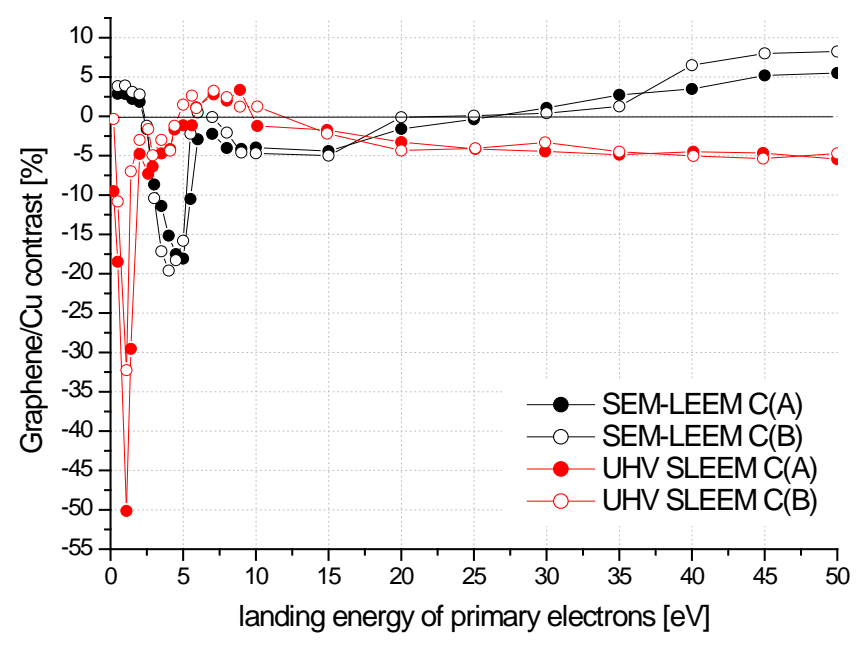

Figure 3. Dependence of the contrast between the graphene and the $\mathrm{Cu}$ substrate on the incident electron energy. Black lines: the contrast calculated from the SEM-LEEM micrographs, red lines: the contrast calculated using the UHV SLEEM micrographs; $\mathrm{C}(\mathrm{A})=$ contrast between the graphene on the grain $\mathrm{A}$ and the grain $\mathrm{A}, \mathrm{C}(\mathrm{B})=$ contrast between the graphene on the grain $\mathrm{B}$ and the grain $\mathrm{B}$ (marked in Fig. 2 (i, i)). 